Signal \& Image Processing : An International Journal (SIPIJ) Vol.4, No.4, August 2013

\title{
Novel Algorithm For SKIN COLOR BASED SEGMENTATION USING MIXTURE OF GMMS
}

\author{
Mokhtar M. Hasan ${ }^{1}$, Pramod K. Mishra ${ }^{2}$ \\ ${ }^{1}$ Computer Science Department, Baghdad University, Iraq \\ ${ }^{1}$ mmwaeli@gmail.com \\ ${ }^{2}$ Computer Science Department, Banaras Hindu University, India \\ ${ }^{2}$ pkmisraegmail.com
}

\begin{abstract}
Image segmentation techniques are considered the main artifact against which the computer can visualize the objects in that scene and for further processing, many hand segmentation techniques are adopted in this direction which consumes the color cue as the enjoinder tools for spotting the skin pixels and non-skin pixels, GMM has been implemented successfully in this area which can tone single color model using single GMM, some shortcoming may come along with this technique, our proposed technique is to combine several color models each one is modeled using GMM and the final model is mixture of GMMs thatcan get rid of the single errors that may occur with single GMM, we have achieved a very high recognition rate when compared with applying single GMM for the pertinent color models, we have adopted four color models which are commonly used for skin color which are normalized RGB which referred as rgb, HSV, $Y U V$ and $L^{*} a * b^{*}$.
\end{abstract}

\section{KEYWORDS}

Segmentation techniques, Gaussian mixture model, GMM, Bayesian decision rule, mixture of GMM, skin color segmentation, color based segmentation, GMM for skin color.

\section{INTRODUCTION}

Vision based hand gesture recognition system methods considered as a natural and intuitive more than glove based methods which require the user to have some kind of wires with a glove to be attached with the hand [1]. Even though these methods provide a high level degree of precise with hand orientation and fingers positions but still obstruct the ease of using natural hand for different applications especially for human computer interaction system (HCI) [2].

Various methods have been addressed the problem of hand gesture interpretation and recognition [1]. For modeling hand gesture in vision based approaches two methods are available appearance based modeling and 3D model modeling based techniques [1][2][3]. Appearance based modeling techniques related with the hand appearance in the input viewed images [1]. 3D model based modeling techniques which can be classified into volumetric models and skeletal models [3]. Volumetric models which usually used in computer graphics and animation [3] are complex and

DOI : 10.5121/sipij.2013.4412 
Signal \& Image Processing : An International Journal (SIPIJ) Vol.4, No.4, August 2013

require huge computations to represent its numerous amounts of volumetric parameters [3]. Skeletal models on the other hand require less parameter for modeling the hand using the 27

bones which represent the degree of freedom (DoF) in human hand [3]. Glove based used for capturing 3D of the hand [1]. In this paper appearance based approach are used for modeling human hand.

Hand segmentation is the preliminary phase for many applications such as pattern recognition [4], traffic control system [5], and surveillance [5]. Since the segmentation is the division of the colored image into meaningful regions to ease the image analysis [6]. One of the efficient characteristics that can be utilized in hand segmentation is skin color [7], where this property is easy to detect and invariant to scale, and rotation changes [8]. The skin color based segmentation classifies the hand image into skin and non-skin color pixels. The best segmentation of skin color pixels should cover various types of skin color such as blackish, brownish [9] under different illumination changes [9].

The tracking hand can be segmented in two ways: the first is by utilizing from tracking information [10] with the help of some techniques such as Kalman filter, and HMM [10],this method needs to initialize the start position of the hand location [10][11]. The second method is to deal with each frame separately and treats each hand pose as a posture [10] in this case the tracking information will be unnecessary. The performance of segmentation process are affected by the representation of the color space used, intricate background, and variant lightings as well [12].

The paper organized as follows. Section 2 presents color space specification. Section 3 presents segmentation techniques. Section 4 discussed Statistical Based Segmentation methods. In Sections 5 the proposed approach are explained with the mathematical model and performance evaluation. Section 6 demonstrates experimental results, finally conclusion are concluded in Section 7.

\section{Color SPaCe SPecification}

Various color spaces are in apply for processing colored images [13]. Since the image acquired from digital camera or video in RGB color model, it is the common color space used for processing colored images [14]. Many color spaces are available for representing the color images, each has different characteristics and they share some properties [9]. The main color spaces are RGB, HSI/ HSV/ HSL, YIQ/ YUV/ YCbCr, and CIE Lab/ CIE Luv [9][15]. The degree of correlations between RGB components is lofty which produce a perceived sensitivity to illumination changes [16], and would hinder the performance of the segmentation process especially for methods that rely on skin color in detection aims [16]. To overcome this obstacle other color spaces are applied on the input images. Since each color space consists of chrominance and luminance components [16], and the luminance component affected with lighting changes. The researchers used to neglect the luminance component and use only the chrominance component [15] for modeling the hand skin color and fitting their models, these color models named normalized color models such as normalized-rg, and HS color models [15] are simple to implement and, easy to calculate, as well as robust to environments lighting changes [16]. 
Signal \& Image Processing : An International Journal (SIPIJ) Vol.4, No.4, August 2013

\subsection{Skin Color Modelling}

Numerous methods prefer to use skin color modeling for regions segmenting [16], since it is invariant to skin position changes such as rotation and scaling [17] as well as easy to apply [17] as aforementioned. The selected color space influences on the modeling of skin color and the specific application as well [16]. However, human skin color might overlaps with other skin color in the complex background and causes misclassification of the region of interest and imposes some limitations on the environments or to the user [18]. Such limitations can be achieved by using non-skin color objects in the background, or some kind of gloves such as instrumented glove, or colored markers [18][19] where the gloves can measure the hand shape [19][20], position [21], and the joint angles [20][21]. Other systems used depth information acquired form special devices such as Time of Flight (ToF) camera [16] or sensor [18] which captures the object's depth information for each pixel and measure the distance using infrared brightness [16]. Statistical methods such Gaussian mixture model GMM [8][15][16] and non-parametric modelling such as histogram based-modeling [16][22] are often used for skin color modeling.

\section{SEgMENTATION TECHNiQUES}

Segmentation process aims to classify the input image into regions by discovering suitable characteristics for these regions to easily recognize the regions of interest [23]. Two properties can be applied to direct the segmentation process [10]; discontinuity, and similarity. Discontinuity refers to sudden change in image intensity which represents the edge of the segmented regions [23], while similarity refers to the similar intensity values between neighboring pixels in the same region [23].

Segmentation techniques can be classified into three main types which are: pixel based, edge based, and region based. The choice of a specific technique related to the complexion of the required application and the surrounding environments [23]. A brief explanation of these techniques is presented in the following subsections.

\subsection{Pixel Based Segmentation}

The simplest segmentations methods is the pixel based known also as point based or thresholding method [23][24]. In this method image pixels are classified according to specific threshold values [24]. Various algorithms are proposed for skin color detection [9], such as piecewise linear classifiers [25], Bayesian classifier [6][26], histogram [27], fuzzy clustering [14], Gaussian classifiers [28][29], histogram based thresholding [13][14][24], Neural Networks NNs [13][30].

\subsection{Edge-Based Segmentation}

Edge based or known as boundary-based segmentation methods [31] indicate the segmenting of the image depending on the edges between the segmented regions [4][13] through finding the connection between edge pixels to form a contour [32]. This can be performed either automatically using some edge detection filters [24], or manually by dragging the mouse to create boundaries between the segmented regions [4][24]. Examples of edge detection filters are Prewitt's filter [24], Laplacian of Gaussian filter [24], watershed segmentation algorithm [4], and canny edge detector [31]. 
Signal \& Image Processing : An International Journal (SIPIJ) Vol.4, No.4, August 2013

\subsection{Region-Based Segmentation}

In this method the image is divided into groups of similar pixels that share some amenities [33]. Region-based methods follow the principle of similarity values of the neighboring pixels in the same region [4] by comparing each pixel with neighboring pixels to determine the region it belongs to according to similarity conditions [4]. The segmentation process in region-based methods uses the feature image rather than the original image, where the feature image is represented by the regions classified by the segmentation process [23], however these methods are affected with the noise [4]. Some region based skin classifications are: region growing, region merging, and region-splitting algorithms [14].

\section{Statistical BaSed Segmentation TechniQueS}

Gaussian Model (GM) and Gaussian Mixture Model (GMM) are statistical methods used widely for skin color segmentation. The mathematical representation for GMM can be noted by the following formula in (1):

$\mathrm{P}(\mathrm{x})=\sum_{\mathrm{j}=1}^{\mathrm{K}} \mathrm{w}_{\mathrm{j}} \cdot \mathrm{N}\left(\mathrm{x} \mid \mu_{\mathrm{j}}, \sum_{\mathrm{j}}\right)$

And $w_{j}$ is the weight of the $\mathrm{j}^{\text {th }}$ mixture.

$\sum_{\mathrm{j}=1}^{\mathrm{K}} \mathrm{w}_{\mathrm{j}}=1 \quad$ and $\quad 0 \leq \mathrm{w}_{\mathrm{j}} \leq 1$

Where $\mu$ is the mean and $\sum$ is the covariance matrix, and $\left|\sum\right|$ its determinant. In [29] two complexion models (YCbCr space and $\mathrm{Nrg}$ space) are integrated using Gaussian distribution with the background model to implement robust hand segmentation method. Gaussian parameters the mean value and covariance matrix are produced by Gaussian model maximum likelihood estimation. When applying GM on YCbCr model some overlapping problem appear because of other skin likely regions in the background, this problem overcomes using Nrg space according to the following conditions [29]:

$r \in[0.33,0.51], g \in[0.28,0.35]$, even. $r>g$

And for any changes in system environments the background model can update the expected value and the variance of color distribution according to the time $t$ at point i.The authors in [34] performed skin segmentation using Gaussian mixture model (GMM) over YCbCr color space. The skin pixels have been modeled using K-means clustering algorithm to initialize the Gaussian parameters, and Expectation Maximization (EM) algorithm to estimate the maximum likelihood of the parameters (Mean, covariance matrix and mixture weight). The system trained with 200 video samples and tested with 98 video samples. The authors in [35] combined two techniques the GMM and histogram-based method to specify the hand. The system trained offline using GMM and tested online using the histogram in real system. Since GMM is sensitive to illumination changes, histogram based method used to overcome this problem and the two methods are integrated. GMM used to model the skin distribution using r-g color space and the histogram used HS color model. Normalized color spaces are used in the proposed system for simplicity and to reduce computations time needed [16]. HSI color model efficient with 
Signal \& Image Processing : An International Journal (SIPIJ) Vol.4, No.4, August 2013

histogram classifier but requires highly computational, and for that reason, look-up table (LUT) used to store the beforehand HSI values and increase the computational speed as well [35]. In article [16] they applied same system proposed by [35] for skin color based model with the depth information acquired from ToF camera [16]. In article [7] they used GMM to estimate the probability of human skin color. K-means algorithm used to initialize Gaussian parameters and EM algorithm used to estimate the parameters, while the number of parameters is determined using Minimum Description Length (MDL) algorithm. Three different color spaces are used in the suggested system: YES, chromatic space and log-opponent models. The number of samples applied in YES color space for training was 144 color image 15,345 skin (in 42 images) pixels and 83,353 non-skin pixels (in 102 images), and for testing was 60 different color images 13,345 skin pixels and 28,669 non-skin pixels. [9] evaluated the performance of Gaussian Model (GM), and Gaussian Mixture Model (GMM), with the histogram based Bayesian classifier and Neural Network $(\mathrm{NN})$. The conclusion finds out that both the latters have promising performance than both Gaussian classifiers. For training the system, the number of samples was 2500 images with 116.6 million for skin pixels and 564.1 million for non-skin pixels, and for testing were 1500 images with 92.8 million skin pixels and 337.7 million for non-skin pixels. The authors in [36] proposed GMM with the modified EM algorithm named as EM-MAP (Maximum a Posterior). The EM-MAP algorithm is pixel labeling procedure that provides a different label for each segmented object in the image using Bayes rule and sequences of prior probability followed by sequence of posterior probability until a convergence stage reached [36]. However the suggest algorithm has shortcoming in deciding some prior parameters like the number of classes, the weights, the mean and variance [36]. These parameters are estimated practically using the histogram of the required image, but the outcome information has high entropy [36]. When the EM-MAP algorithm reached to the convergence stage, stable level in entropy posterior probability is achieved after some iteration [36]. However this algorithm suitable for simple images but inapplicable on real images that depends on the features for modeling the segmented images [36].

\section{Proposed Algorithm}

In this paper we built a model that combined four color spaces for skin color based segmentation process. The most four color spaces commonly used for skin color segmentation are RGB, HSV, $\mathrm{YCbCr}$, and $\mathrm{L}^{*} \mathrm{a}^{*} \mathrm{~b}^{*}$ are utilized in this work. Since each color space has weak features when applied separately for skin color distribution, we proposed to integrate these color spaces into a single model by discarding unhelpful features of each color space. Each color space has been modeled using a single GMM, and the resultant models are melted down into one superior model with a weight value for each GMM that represent the classification rate. The output model named as Mixture of GMM (MiGMM) that provesand ensures the high performance as compared with each single GMM separately.

\subsection{Mathematical Model}

The proposed model has the following mathematical representation as seen in Equation 4.

$\mathcal{P}(\mathrm{c} \mid$ skin $)=\sum_{\mathrm{m}=1}^{\mathrm{M}} \mathrm{W}_{\mathrm{m}} \mathrm{P}\left(\mathrm{c} ; \mu_{\mathrm{m}}, \sum_{\mathrm{m}}\right)$ 
Signal \& Image Processing : An International Journal (SIPIJ) Vol.4, No.4, August 2013

Where $\mathrm{c}, \mathrm{M}, \mathrm{m}$ and $\mathrm{P}\left(\mathrm{c} ; \mu_{\mathrm{m}}, \sum_{\mathrm{m}}\right)$ represent the same parameters as in Equation $1, \mathrm{~W}_{\mathrm{m}}$ represents the weight of $m$ component which satisfies the same condition of Equation 2 that has the value of one as its grand summation.

$\mathrm{W}_{\mathrm{m}}$ represents the missing value in the suggested MiGMM model, and can be found by calculating the classification rate, where $\mathrm{W}_{\mathrm{m}}$ summarizes the classification rate of the algorithm. This metric parameter is calculated as seen in Equation 5.

$\mathrm{W}_{\mathrm{m}}=\frac{\mathrm{CR}_{\mathrm{m}}}{\sum_{\mathrm{i}=1}^{\mathrm{M}} \mathrm{CR}_{\mathrm{i}}}, \forall \mathrm{m}=1,2,3, \ldots, \mathrm{M}$

The next section explains the classification rate along with other metric parameters.

\subsection{Performance Evaluation}

In this work three metric parameters are implemented as in [25] which are CDR, FDR, and CR. In the proposed model 100 images are used for testing with their ground truth pictures to build a fair ground when evaluation the performance of the model, the metric parameters used are as follows [25]:

Correct Detection Rate (CDR):The percentage of the pixels that are classified correctly by the algorithm as skin pixels.

False Detection Rate (FDR):The percentage of the pixels that are classified wrongly by the algorithm as non-skin pixels.

Classification Rate (CR): The number of skin pixels that classified correctly by the algorithm and ground truth is divided by whichever maximum of each number of skin pixels classified by the algorithm and number of skin pixels classified by the ground truth.

In mathematical representation, Equations 6, 7 and 8 correspond to each of CDR, FDR, and CR respectively.

$$
\begin{aligned}
& \mathrm{CDR}=\frac{\mathrm{C}_{\mathrm{s}}^{\mathrm{a}}}{\mathrm{T}_{\mathrm{s}}^{\mathrm{g}}} * 100 \% \\
& \mathrm{FDR}=\frac{\mathrm{W}_{\mathrm{ns}}^{\mathrm{a}}}{\mathrm{T}_{\mathrm{ns}}^{\mathrm{g}}} * 100 \% \\
& \mathrm{CR}=\frac{\mathrm{C}_{\mathrm{s}}^{\mathrm{a}}}{\max \left(\mathrm{T}_{\mathrm{s}}^{\mathrm{a}}, \mathrm{T}_{\mathrm{s}}^{\mathrm{g}}\right)} * 100 \%
\end{aligned}
$$

Where $C_{s}^{a}$ represents the total number of pixels that classified correctly as skin pixels by the proposed algorithm, $\mathrm{T}_{\mathrm{s}}^{\mathrm{g}}$ represents the total number of skin pixels that are classified by the ground truth, $W_{\text {ns }}^{\mathrm{a}}$ represents the total number of pixels that are classified wrongly as non-skin pixels by the algorithm, $\mathrm{T}_{\mathrm{ns}}^{\mathrm{g}}$ is the total number of non-skin pixels that are classified by the ground truth, and finally, $\mathrm{T}_{\mathrm{s}}^{\mathrm{a}}$ represents the total number of skin pixels that classified by the algorithm. 
Signal \& Image Processing : An International Journal (SIPIJ) Vol.4, No.4, August 2013

\section{EXPERIMENTAL RESULTS}

After segmenting skin color pixels and modeling the distribution, the system trained using 757.883 skin pixels. the background color distribution have trained also using the same previously mentioned model, to make a good and fair comparative study about the existence of background color model or not, however, the background color distribution models have trained with 341.924 color pixels.

Equation 8 has been adopted to calculate the weights of each GMM which corresponds to a single color model, the weight list as calculated is: $W=\{0.250438924,0.249292548,0.25049777$, 0.249770759 \} for each of the rgb, HSV, YCbCr and L*a*b* respectively.

As earlier explained, the testing performed using 100 input images, Table 1 shows the calculation of metric parameters for segmentation operation using single color GMM model as well as our proposed mixture of all of them.

We have applied two cases for training and testing operations, the first case is by adopting the skin pixels only for skin color modelling and the corresponding foreground model has been fitted, in the second case, the background pixels are used to feed the model data along with previously foreground model, in the first case the model is called F model and in the second case the model is called FB model as noted in mentioned table.

Table 1. Metric parameters for the proposed and selected color models.

\begin{tabular}{l|l|l|l|l|l|l|l|l|l|l}
\hline \multirow{2}{*}{$\begin{array}{l}\text { parame } \\
\text { ter }\end{array}$} & \multicolumn{2}{|c|}{ MiGMM } & \multicolumn{2}{c|}{$\begin{array}{c}\text { GMMof } \\
\text { rgb }\end{array}$} & \multicolumn{2}{c|}{$\begin{array}{c}\text { GMM of } \\
\text { HSV }\end{array}$} & \multicolumn{2}{|c|}{$\begin{array}{c}\text { GMM of } \\
\text { YCbCr }\end{array}$} & \multicolumn{2}{|c}{$\begin{array}{c}\text { GMM of } \\
\text { L*a*b* }\end{array}$} \\
\cline { 2 - 11 } & F (\%) & FB (\%) & F (\%) & FB (\%) & F (\%) & FB (\%) & F (\%) & FB (\%) & F (\%) & $\begin{array}{c}\text { FB } \\
(\%)\end{array}$ \\
\hline CDR & 99.0149 & 99.1993 & 99.6525 & 97.7338 & 97.9378 & 99.5810 & 98.4020 & 99.7922 & 99.3646 & 99.8386 \\
\hline FDR & 0.1291 & 0.0405 & 0.4416 & 0.0092 & 0.1156 & 0.7039 & 0.1887 & 1.0098 & 0.3703 & 0.7590 \\
\hline CR & 99.0149 & 99.1993 & 98.9036 & 97.7338 & 97.9378 & 98.2625 & 98.4020 & 97.5311 & 99.0761 & 98.1336 \\
\hline Average & 99.300 & 99.452 & 99.371 & 98.486 & 98.586 & 99.046 & 98.871 & 98.771 & 99.356 & 99.071 \\
\hline
\end{tabular}

As seen by latter table, our proposed algorithm secured the highest recognition rates as compared with each of other GMM models separately that gives $99.3 \%$ for foreground modelling and $99.452 \%$ for both foreground and background modelling.

Pictorial speaking, we have prepared Figure 1 that shows an ocular comparison for application of skin color segmentation using the aforementioned skin color models.

\section{CONCLUSION}

Segmentation operation is the partitioning of input image or input scene into meaningful objects each can be treated and processed by the computer in automated way separately, those objects are discriminated by their salient color, skin color plays major role for the successful of the segmentation operation since its color is discriminated and be separated easily as compared with other object's color that may fall in the same scene, from this point a plenty of color based segmentation techniques have been emitted and took their place in the practical fields. 
Signal \& Image Processing : An International Journal (SIPIJ) Vol.4, No.4, August 2013

We have suggested combining several color models each color model has been toned using single GMM and the resulted model is the mixture of resulted GMMs, we have achieved high recognition rates by the testimony of three metric parameters by comparing the proposed model and each single GMM.

The adopted components for each color model can be summarized as follows: $\mathrm{r}$ and $\mathrm{g}$ components for rgb color model (normalized RGB), $\mathrm{H}$ and $\mathrm{S}$ components for $\mathrm{HSV}$ color model, $\mathrm{Cb}$ and $\mathrm{Cr}$ for $\mathrm{YCbCr}$ color model, and finally; $\mathrm{a}^{*}$ and $\mathrm{b}^{*}$ for $\mathrm{L}^{*} \mathrm{a}^{*} \mathrm{~b}^{*}$ color model, the main reason behind this selection is to reduce the color space dimension from 3D dimension to $2 \mathrm{D}$ dimension for more easy modelling and also because the illumination component can be neglected to form more robust color based segmentation technique that can act against various illumination and brightness change, just the components that represent the color itself can be modelled which called chrominance components.

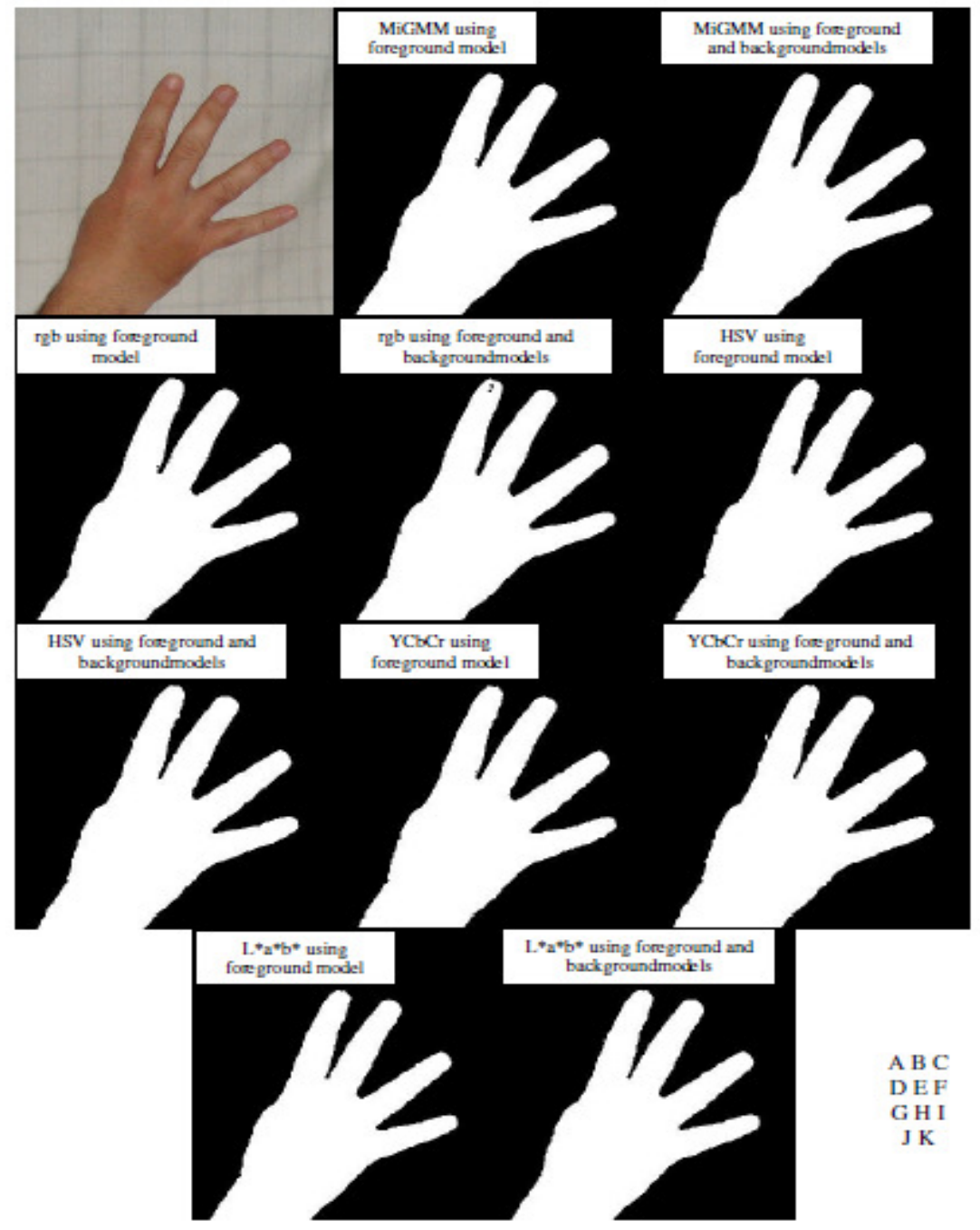

A: original input image for testing.B, D, F, H, J: skin segmentation using foreground model only for MiGMM, rgb, HSV, YCbCr, and L*a*b* respectively.C, E, G, I, K: skin segmentation using foreground and background models for MiGMM, rgb, HSV, YCbCr, and $\mathrm{L}^{*} \mathrm{a} * \mathrm{~b} *$ respectively.

Figure 1: Comparison of skin color segmentation using different adopted techniques. 
Signal \& Image Processing : An International Journal (SIPIJ) Vol.4, No.4, August 2013

\section{REFERENCES}

[1] Xiaoming Yin\& Ming Xie, (2001) "Hand Gesture Segmentation, Recognition and Application", IEEE International Symposium on Computational Intelligence in Robties and Automation, pp. 438- 443, Canada.

[2] S. Mitra, \& T. Acharya, (2007) "Gesture Recognition: A Survey", IEEE Transactions on systems, Man and Cybernetics, Part C: Applications and reviews, Vol. 37, No. 3, pp. 311-324. doi: 10.1109/TSMCC.2007.893280.

[3] Pavlovic, V. I., Sharma, R.\& Huang, T. S. (1997) "Visual Interpretation of Hand Gestures for Human- Computer Interaction: A Review", IEEE Transactions On Pattern Analysis And Machine Intelligence, Vol.19, No. 7, pp. 677- 695, doi; 10.1109/34.598226.

[4] Yu-Hsiang Wang, "Tutorial: Image Segmentation", Graduate Institute of Communication Engineering National Taiwan University, Taipei, Taiwan.

[5] Wikipedia website.

[6] P.Daniel Ratna Raju, G.Neelima\& K.Prasada Rao, (2011) "Image segmentation-MR Images Segmentation with A Modified Gaussian Mixture Model", International Journal of Computer Science and Information Technologies (IJCSIT), Vol. 2, No. 6 , pp. 2573-2578.

[7] Demir Gokalp, "Learning Skin Pixels in Color Images Using Gaussian Mixture".

[8] Paola Campadelli, Francesco Cusmai\& Raffaella Lanzarotti, (2003) "A Color-Based Method For Face Detection".

[9] Son Lam Phung, Abdesselam Bouzerdoum\& Douglas Chai, (2005) "Skin segmentation using color pixel classification: analysis and comparison", IEEE Transactions on Pattern Analysis and Machine Intelligence, Vol. 27, No. 1, pp. 148-154.

[10] Mokhtar M. Hasan\& Pramod K. Mishra, (2012) "Hand Gesture Modeling and Recognition using Geometric Features: A Review", Canadian Journal on Image Processing and Computer Vision Vol. 3, No. 1.

[11] Ruiduo Yang\& Sudeep Sarkar, (2006) "Gesture Recognition using Hidden Markov Models from Fragmented Observations", IEEE Conference on Computer Vision and Pattern Recognition (CVPR'06), Vol. 1. Doi: 10.1109/CVPR.2006.126

[12] Sergio 'Alvarez, David F. Llorca, Gerard Lacey\&Stefan Ameling, (2010) "Spatial Hand Segmentation Using Skin Colour And Background Subtraction", Trinity College Dublin's Computer Science Technical Report, Dublin.

[13] L. Lucchese \& S. K. Mitra, (2001) "Color Image Segmentation: A State-of-the-Art Survey", Indian national science academy, Vol. 67, No. 2, pp.207-221. Doi: 10.1.1.84.4896

[14] Władysław Skarbek \& Andreas Koschan, (1994) "Colour Image Segmentation: A Survey", Berlin, Germany.

[15] Jean-Christophe Terrillon, Mahdad N. Shirazi, Hideo Fukamachi\& Shigeru Akamatsu, (2000) "Comparative Performance of Different Skin Chrominance Models and Chrominance Spaces for the Automatic Detection of Human Faces in Color Images", IEEE the Fourth International Conference on Automatic Face and Gesture Recognition (FG '00).

[16] Zhan Gao, "Appearance-based Hand Gesture Detection".

[17] E. Stergiopoulou\& N. Papamarkos, (2009) "Hand gesture recognition using a neural network shape fitting technique", Elsevier, Engineering Applications of Artificial Intelligence, vol. 22 pp. 11411158.doi:10.1016/j.engappai.2009.03.008.

[18] S. E. Ghobadi, O. E. Loepprich, K. Hartmann \& O. Loffeld, (2007) "Hand Segmentation Using 2D/3D Images", conference of Image and Vision Computing New Zealand, pp. 64-69, New Zealand.

[19] Sudeep Sarkar, Barbara Loeding, Ruiduo Yang, Sunita Nayak\& Ayush Parashar, (2011) "Segmentation-robust Representations, Matching, and Modeling for Sign Language", IEEE Computer Society Conference on Computer Vision and Pattern Recognition Workshops (CVPRW), pp. 13-19. Doi: 10.1109/CVPRW.2011.5981695

[20] Thiago R. Trigo \& Sergio Roberto M. Pellegrino, (2010) "An Analysis of Features for Hand-Gesture Classification", 17th International Conference on Systems, Signals and Image Processing (IWSSIP 2010), pp. 412- 415. 
Signal \& Image Processing : An International Journal (SIPIJ) Vol.4, No.4, August 2013

[21] Vaishali S. Kulkarni\& S.D.Lokhande, (2010) "Appearance Based Recognition of American Sign Language Using Gesture Segmentation”, International Journal on Computer Science and Engineering (IJCSE), Vol. 2, No. 3, pp. 560-565.

[22] Michael J. Jones\& James M. Rehg, (1999) "Statistical Color Models with Application to Skin Detection", IEEE Conference on Computer Vision and Pattern Recognition. Vol. 1. Doi; 10.1109/CVPR.1999.786951

[23] Krishna Kant Singh \&Akansha Singh, (2010) "A Study of Image Segmentation Algorithms For Different Types of Images", International Journal of Computer Science Issues IJCSI, Vol. 7, No. 5.

[24] Chapter 4 Segmentation.

[25] Francesca Gasparini\& Raimondo Schettini, (2006) "Skin Segmentation Using Multiple Thresholding", In Internet imaging VII, IS and T/SPIE, pp. 60610F-1-60610F-8. SPIE.

[26] Vladimir Vezhnevets, Vassili Sazonov\& Alla Andreeva, (2003) "A Survey on Pixel-Based Skin Color Detection Techniques", IN PROC. GraphiCon-2003.

[27] Yikai Fang, Kongqiao Wang, Jian Cheng\& Hanqing Lu, (2007) "A Real-Time Hand Gesture Recognition Method", IEEE, ICME, pp. 995-998.

[28] Cheng-Chin Chiang, Wen-Kai Tai, Mau-Tsuen Yang, Yi-Ting Huang\& Chi-Jaung Huang, (2003) "A novel method for detecting lips, eyes and faces in real time", Elsevier Real-Time Imaging, Vol. 9, pp. 277-287. Doi: 10.1016/j.rti.2003.08.003.

[29] Shuying Zhao, Wenjun Tan, Shiguang Wen, \& Yuanyuan Liu, (2008) "An Improved Algorithm of Hand Gesture Recognition under Intricate Background", the First International Conference on Intelligent Robotics and Applications (ICIRA 2008): Part I. Springer-Verlag Berlin Heidelberg, pp. 786-794. Doi:10.1007/978-3-540-88513-9_85

[30] M. K. Viblis\& K. J. Kyriakopoulos, (2000) "Gesture Recognition: The Gesture Segmentation Problem", Journal of Intelligent and Robotic System, Vol. 28, pp. 151-158. Doi: 10.1023/A:1008101200733

[31] Hassana Grema Kaganami, Zou Beiji, "Region-Based Segmentation versus Edge Detection", IEEE Fifth International Conference on Intelligent Information Hiding and Multimedia Signal Processing, pp. 1217-1221, 2009. DOI: 10.1109/IIH-MSP.2009.13

[32] Fritz Albregtsen, (2010) "Region \& Edge Based Segmentation".

[33] http://homepages.inf.ed.ac.uk/rbf/CVonline/LOCAL_COPIES/RAMANI1/node19.html

[34] Mahmoud Elmezain, Ayoub Al-Hamadi, J"org Appenrodt, \& Bernd Michaelis, (2008) "A Hidden Markov Model-Based Isolated and Meaningful Hand Gesture Recognition", World Academy of Science, Engineering and Technology, Vol. 41.

[35] M. Van den Bergh, E. Koller-Meier, F. Bosch'\& L. Van Gool, (2009) "Haarlet-based Hand Gesture Recognition for 3D Interaction", Workshop on Application of Computer Vision (WACV), pp. 1-8, doi: 10.1109/WACV.2009.5403103

[36] Rahman Farnoosh\& Behnam Zarpak, (2008) "Image Segmentation Using Gaussian Mixture Model", International Journal of Engineering Science (IUST), pp. 29-32, Vol. 19, No.1. 